\title{
UNIQUENESS THEORY FOR LAPLACE SERIES $\left({ }^{1}\right)$
}

\author{
BY \\ WALTER RUDIN

\section{INTRODUCTION}

1.1. Spherical surface harmonics. Let $S$ denote the surface of the unit sphere, whose center is at the origin $O$ of a system of Cartesian coordinates $x, y, z$. Let $P$ denote a point on $S$, and let $(r, P)$ be the point which lies on the vector $O P$, at a distance $r$ from $O$. The function $Y_{n}(P)$, defined on $S$, is said to be a spherical surface harmonic of degree $n$ if the function

$$
H_{n}(x, y, z)=H_{n}(r, P)=r^{n} Y_{n}(P)
$$

is a homogeneous harmonic polynomial in $x, y, z$. The orientation of the axes is immaterial, since the harmonic property and the degree of $H_{n}$ are preserved under a rotation of the coordinate system.

Spherical surface harmonics of different degree are orthogonal to each other, that is $[1 ; 144]$,

$$
\iint_{S} Y_{n}(P) Y_{m}(P) d P=0 \quad(m \neq n) .
$$

1.2. Laplace series. Suppose $f \in L$ on $S$. With $f(P)$ there is associated a sequence of spherical surface harmonics

$$
Y_{n}(P)=\frac{2 n+1}{4 \pi} \iint_{S} f(Q) P_{n}(\cos P Q) d Q \quad(n=0,1,2, \cdots),
$$

where $P Q$ denotes the shorter great circle arc on $S$ between $P$ and $Q$, and $P_{n}(x)$ is the Legendre polynomial defined $[1 ; 15]$ by the generating function

$$
\left(1-2 r x+r^{2}\right)^{-1 / 2}=\sum_{n=0}^{\infty} P_{n}(x) r^{n}
$$
write

The series $\sum_{n=0}^{\infty} Y_{n}(P)$ defined by (1.2.1) is the Laplace series of $f(P)$. We

$$
f(P) \sim \sum_{n=0}^{\infty} Y_{n}(P)
$$

Presented to the Society, April 2, 1949; received by the editors July 27, 1949.

(1) Submitted in partial fulfillment of the requirements for the degree of Doctor of Philosophy in the Graduate School of Arts and Sciences of Duke University (see also [6]). The author wishes to acknowledge his indebtedness to Professor J. J. Gergen, under whose direction the thesis was written. Numbers in brackets refer to the bibliography at the end of the paper. 
The problem we shall consider (the so-called "uniqueness problem") is to determine conditions under which a given series of spherical surface harmonics is a Laplace series.

Uniqueness properties have been studied for a number of orthogonal systems in one variable, with finite ranges of orthogonality, in particular for trigonometric series (starting with Riemann), Legendre series [3], and Bessel series ([9] contains numerous references). The present paper seems to be the first in which the uniqueness problem is considered for a system which is orthogonal over a two-dimensional set $\left({ }^{2}\right)$. Our main results (Theorems 2.6 and 2.7) are of a nature similar to theorems of Zygmund [8;284] and Verblunsky $[7 ; 462]$ on the uniqueness of Fourier series.

Theorem 3.9 is an analogue of Theorem I of [6]. From Theorem 3.9 we derive Theorem 4.2, which is fundamental for our proof of the uniqueness theorems.

\section{Statement of Main Results}

2.1. Notation. For $P$ on $S$, and $0<h<\pi$, we let $C(P, h)$ denote the circle of intersection of $S$ and the sphere of radius $2 \sin 2^{-1} h$ about $P$ as center. By the interior $I(P, h)$ of $C(P, h)$ we mean the domain on $S$ which contains $P$ and is bounded by $C(P, h)$. If $F \in L$ on $C(P, h)$, we put

$$
\Delta_{h} F(P)=\frac{1}{2 \pi \sin h} \int_{C(P, h)} F(Q) d s_{Q}-F(P) .
$$

If there exists $\delta>0$ such that $F \in L$ on $C(P, h)$ for $0<h<\delta$, we define the generalized Laplacian of $F(P)$ by

$$
\psi F(P)=\lim _{h \rightarrow 0} \Delta_{h} F(P) / \sin ^{2} 2^{-1} h,
$$

provided the limit exists. The upper and lower generalized Laplacians $\psi^{*} F(P)$ and $\psi_{*} F(P)$ are defined likewise, with lim sup and lim inf in place of lim.

The operator $\psi$ is an analogue of the generalized second differential operator $D^{2}[10 ; 270]$, and of the operator $\Lambda[6]$. The special significance of $\psi$ for our purpose is shown by (5.1.1).

2.2. Definition. We say that the series $\sum_{n=0}^{\infty} Y_{n}(P)$ of spherical surface harmonics is of class $K$ if the series $-\sum_{n=1}^{\infty} Y_{n}(P) / n(n+1)$ is the Laplace series of a function continuous on $S$.

2.3. Definition. Suppose the series $\sum_{n=0}^{\infty} Y_{n}(P)$ is of class $K$. Let $F(P)$ be the continuous function whose Laplace series is $-\sum_{n=1}^{\infty} Y_{n}(P) / n(n+1)$

(2) After publication of an abstract of the present paper, the author received from Professor Plancherel a reprint of a paper (Bull. Sci. Math. (2) vol. 43 (1919)) in which he proved the following theorem. If (1) $\sum Y_{n}(P)$ converges to $f(P)$ on $S-E$, where $E$ is a reducible set, (2) $f \in L^{2}$ on $S$, (3) $f(P)$ is bounded on $S$, except possibly in neighborhoods of points of $E$, (4) $-\sum Y_{n}(P) / n(n+1)$ converges uniformly on $S$, then $f(P) \sim \sum Y_{n}(P)$. This result is a corollary of Theorem 2.7 . 
(since two functions having the same Laplace series are equal p. p. on $S, F$ is uniquely determined). We say that the functions $Y_{0}+\psi^{*} F(P)$ and $Y_{0}$ $+\psi_{*} F(P)$ are the upper and lower Riemann sums, respectively, of the series $\sum_{n=0}^{\infty} Y_{n}(P)$.

If $\psi F$ exists finitely at a point $M$ on $S$, we say that the series is Riemann summable at the point $M$ to the value $Y_{0}+\psi F(M)$. $270]$.

An analogous definition for trigonometric series may be found in $[10$;

2.4. Definition. Given the series $\sum_{n=0}^{\infty} Y_{n}(P)$. The functions

$$
f^{*}(P)=\lim \sup _{r \rightarrow 1} \sum_{n=0}^{\infty} Y_{n}(P) r^{n}, \quad f_{*}(P)=\liminf _{r \rightarrow 1} \sum_{n=0}^{\infty} Y_{n}(P) r^{n}
$$

are called the upper and lower Poisson sums, respectively, of the given series. If $f^{*}(M)=f_{*}(M)$, and the common value is finite, the given series is said to be Poisson summable at the point $M$ to this common value.

2.5. Definition. A closed set $Z$ on $S$ is said to be of capacity zero on $S$ if $Z$ is a proper subset of $S$ and if the stereographic image of $Z$ in a tangent plane, with center of projection in $S-Z$, is a plane set of capacity zero.

This definition will be justified in 3.4 .

We now state our main results.

2.6. TheOREM. Given a series $\sum_{n=0}^{\infty} Y_{n}(P)$ of spherical surface harmonics. Let $Z$ be a closed set of capacity zero on $S$. Suppose

(i) the given series is of class $K$;

(ii) $\psi^{*} F(P)>-\infty ; \psi_{*} F(P)<+\infty$ on $S-Z$, where $F(P)$ is the continuous function whose Laplace series is $-\sum_{n=1}^{\infty} Y_{n}(P) / n(n+1)$;

(iii) there exists a function $y(P)$, defined on $S, y \in L$ on $S$, such that $y(P)$ $\leqq \psi^{*} F(P)$ for $P$ on $S$.

Then the given series is Riemann summable almost everywhere on $S$ and is the Laplace series of its Riemann sum.

2.7. Theorem. Given a series $\sum_{n=0}^{\infty} Y_{n}(P)$ of spherical surface harmonics, having $f^{*}(P)$ and $f_{*}(P)$ as upper and lower Poisson sums, respectively. Let $Z$ be a closed set of capacity zero on $S$. Suppose

(i) the given series is of class $K$;

(ii) $f^{*}(P)$ and $f_{*}(P)$ are finite on $S-Z$;

(iii) there exists a function $y(P)$, defined on $S, y \in L$ on $S$, such that $y(P)$ $\leqq f_{*}(P)$ for $P$ on $S$.

Then the given series is Poisson summable almost everywhere on $S$ and is the Laplace series of its Poisson sum.

2.8. In the following two corollaries the word "summable" stands for "Poisson summable" or "Riemann summable" or "Cesàro summable" or "convergent." 
Corollary I. If the series $\sum Y_{n}(P)$ is of class $K$ and is summable to zero on $S$, except possibly on a closed set of capacity zero, then the series vanishes identically.

This is an immediate consequence of 2.6 and 2.7.

CoRollary II. If the two series $\sum Y_{n}(P)$ and $\sum Y_{n}^{\prime}(P)$ are of class $K$ and if they are summable to the same function $f(P)$ (where it is not necessary that $f \in L$ on $S$ ) except possibly on a closed set of capacity zero, then the two series are identical.

Proof. We apply Corollary I to the series $\sum\left(Y_{n}(P)-Y_{n}^{\prime}(P).\right)$.

\section{HARMONIC AND SUBHARMONIC FUNCTIONS ON $S$}

Harmonic and subharmonic functions play the same role in our investigation as do linear and convex functions in the corresponding treatment for trigonometric series. To define harmonic functions on $S$ we use the analogue of the Gaussian mean value property.

3.1. Definition. A function $F(P)$ is said to be harmonic in a domain $D$ on $S$ if $F(P)$ is continuous in $D$ and if $\Delta_{h} F(P)=0$ for every circle $C(P, h)$ such that $C(P, h)+I(P, h) \subset D$.

3.2. Lemma. Let $C=C(M, h)$ be a circle on $S$. If $F(P)$ and $G(P)$ are harmonic in $I=I(M, h)$ and continuous on $C+I$, and if $G(P)=F(P)$ on $C$, then $G(P)=F(P)$ in $I$.

Proof. Assume that $F>G$ at some point of $I$. Then the function $H(P)$ $=F(P)-G(P)$, which is harmonic in $I$, and which vanishes on $C$, has a positive maximum in $I$. Let $E$ be the closed subset of $I$ on which $H$ attains its maximum. Let $Q$ be a boundary point of $E$, and choose $k>0$ such that $C(Q, k) \subset I$. Then $\Delta_{k} H(Q)<0$, which is a contradiction. The assumption that $F<G$ at some point of $I$ leads to a contradiction in the same manner.

3.3. Theorem. Let $F(P)$ be defined in a domain $D$ on $S$. Let $\Pi$ be a tangent plane of $S$. Map $S$ stereographically onto $\Pi$, taking $P$ into $P^{\prime}, D$ into a finite domain $D^{\prime}$. Put $F^{\prime}\left(P^{\prime}\right)=F(P)$. Then $F(P)$ is harmonic in $D$ if and only if $F^{\prime}\left(P^{\prime}\right)$ is harmonic in $D^{\prime}$.

Proof. Let $\Pi$ be tangent to $S$ at the point $A$. Suppose first that $F^{\prime}\left(P^{\prime}\right)$ is harmonic in $D^{\prime}$. We pick arbitrarily a circle $C=C(M, h)$ on $S$, such that $C(M, h)+I(M, h) \subset D$. Let $C$ map into the circle $C^{\prime}$ of radius $r$ and center $N^{\prime}$. Let $Q$ on $C$ map into $Q^{\prime}$. By Poisson's formula,

$$
F^{\prime}\left(M^{\prime}\right)=\frac{1}{2 \pi r} \int_{C^{\prime}} \frac{r^{2}-t^{2}}{r^{2}-2 r t \cos \gamma+t^{2}} F^{\prime}\left(Q^{\prime}\right) d s_{Q^{\prime}},
$$

where $t=N^{\prime} M^{\prime}, \gamma=\Varangle A N^{\prime} Q^{\prime}$. We have to show that 


$$
F(M)=\frac{1}{2 \pi \sin h} \int_{C} F(Q) d s_{Q}
$$

Noting that $d s_{Q}=\cos ^{2} 2^{-1} A Q d s_{Q^{\prime}}$, and expressing $r, t$, and $A Q$ in terms of $h, A M$, and $\gamma$, we verify that the right members of (3.3.1) and (3.3.2) are equal, which proves (3.3.2).

Suppose next that $F(P)$ is harmonic in $D$. Let $I^{\prime}$ be the interior of a circle $C^{\prime}$ such that $C^{\prime}+I^{\prime} \subset D^{\prime}$. Since $F^{\prime}\left(P^{\prime}\right)$ is continuous in $D^{\prime}$, there exists a function $G^{\prime}\left(P^{\prime}\right)$, harmonic in $I^{\prime}$, continuous on $C^{\prime}+I^{\prime}$, and such that

$$
G^{\prime}\left(P^{\prime}\right)=F^{\prime}\left(P^{\prime}\right) \quad\left(P^{\prime} \text { on } C^{\prime}\right) .
$$

Applying the first part of the theorem, we see that $G^{\prime}\left(P^{\prime}\right)$ maps into a function $G(P)$ which is harmonic in the interior $I$ of a circle $C$ on $S$, continuous on $I+C$, and such that

$$
G(P)=F(P) \quad(P \text { on } C) .
$$

By Lemma 3.2 we therefore have

$$
G(P)=F(P)
$$

Hence

$$
G^{\prime}\left(P^{\prime}\right)=F^{\prime}\left(P^{\prime}\right)
$$$$
\left(P^{\prime} \text { in } I^{\prime}\right) \text {, }
$$

which proves that $F^{\prime}\left(P^{\prime}\right)$ is harmonic in $D^{\prime}$.

3.4. The only functions which are involved in the definition of plane sets of capacity zero [6; Definition 2.3] are harmonic. Hence Theorem 3.3 shows that Definition 2.5 is independent of the tangent plane chosen, as long as the center of projection is in $S-Z$ (otherwise the image set is unbounded).

3.5. Definitron. Let $R$ denote a domain on $S$ whose closure $\bar{R}$ is a proper subset of $S$. Choose a point in $S-\bar{R}$ as center of a stereographic projection, mapping $R$ into a bounded plane domain $R^{\prime}$, whose Green's function is $g^{\prime}\left(P^{\prime}, Q^{\prime}\right)$. (For our purpose it is sufficient to restrict ourselves to domains $R$ which map into Dirichlet domains $R^{\prime}$.) Put $g(P, Q)=g^{\prime}\left(P^{\prime}, Q^{\prime}\right)$. We call $g(P, Q)$ the Green's function of $R$.

Let $A$ denote a point in $S-\bar{R}$. Considering $g(P, Q)$ as a function of $Q$, with $P$ a fixed point in $R$, we have

$$
g(P, Q)=\log \frac{\sin 2^{-1} A P \sin 2^{-1} A Q}{\sin 2^{-1} P Q}+h(P, Q),
$$

where $h(P, Q)$ is harmonic in $R$. The first function on the right of (3.5.1) has a logarithmic singularity at $Q=P$, and is harmonic in $R-P$ (this may be verified by applying 4.4 ).

Let $P N$ be a diameter of $S$. If $N \in S-\bar{R}$, we can take $N$ for $A$ in (3.5.1), and obtain 


$$
g(P, Q)=\log \cot 2^{-1} P Q+h(P, Q) .
$$

Whether $N \in S-\bar{R}$ or not, $g(P, Q)$ is of the form (3.5.2) in a neighborhood of $P$, since

$$
\log \frac{\sin 2^{-1} A P \sin 2^{-1} A Q}{\sin 2^{-1} P Q}=\log \cot 2^{-1} P Q+\log \frac{\sin 2^{-1} A P \sin 2^{-1} A Q}{\cos 2^{-1} P Q}
$$

and the second function on the right is harmonic in a neighborhood of $P$.

3.6. Definition. A function $u(P)$ is said to be subharmonic in a domain $D$ on $S$ if

(a) $u(P)$ is upper semi-continuous in $D$,

(b) $u(P)<+\infty$ in $D$,

(c) $u(P)$ is finite on a dense set in $D$,

(d) for every domain $G$ with boundary $\Gamma$, such that $G+\Gamma \subset D$, the inequality $u(P) \leqq H(P)$ on $\Gamma$ implies $u(P) \leqq H(P)$ in $G$ for every function $H(P)$ which is harmonic in $G$ and continuous on $G+\Gamma$.

This definition is the precise analogue of Riesz's definition $[5 ; 333]$ of a subharmonic function in the plane.

3.7. From Definition 3.6 and Theorem 3.3 we infer that $u(P)$ is subharmonic in a domain $D$ on $S$ if and only if $u(P)$ maps stereographically into a function $u^{\prime}\left(P^{\prime}\right)$, subharmonic in the plane domain $D^{\prime}$.

3.8. Since the definitions of harmonic and subharmonic functions in domains on $S$ are entirely analogous to the definitions of harmonic and subharmonic functions in the plane, it is clear that we can parallel every step of [6] almost word for word. For $g(P, Q)$ we use the local representation (3.5.2). In this way we obtain the following analogue of Theorem I of [6]:

3.9. Theorem. Let $F(P)$ be continuous in the domain $D$ on $S$. Suppose

(i) $\psi^{*} F(P)>-\infty, \psi_{*} F(P)<+\infty$ for $P$ in $D-D \cdot Z$ where $Z$ is a closed set of capacity zero on $S$;

(ii) there exists a function $y(P)$, defined in $D$, such that $y \in L$ on every closed subset of $D$, and such that $y(P) \leqq \psi^{*} F(P)$ in $D$.

Then

(a) $\psi F(P)$ exists at almost all points $P$ of $D$;

(b) $\psi F \in L$ on every closed subset of $D$;

(c) at all points $P$ of $D$ for which

$$
\iint_{I(P, h)}\left|\psi F(Q) \log \cot 2^{-1} P Q\right| d Q<+\infty
$$

for some $h>0$, we have

$$
F(P)=-\frac{1}{2 \pi} \iint_{R} \psi F(Q) g(P, Q) d Q+H(P),
$$


where $R$ is any domain whose closure is a proper subset of $D$, and which contains $P$; $g(P, Q)$ is Green's function for $R$, and $H$ is harmonic in $R$ and assumes the boundary values of $F$. Moreover, the condition (3.9.1) is satisfied at almost all points $P$ of $D$.

If we take the circular domain $I(P, h)$ for $R$, (3.9.2) becomes, by (3.5.2) and Definition 3.1,

$$
\begin{aligned}
F(P)= & -\frac{1}{2 \pi} \iint_{I(P, h)} \psi F(Q) \log \left(\cot 2^{-1} P Q \tan 2^{-1} h\right) d Q \\
& +\frac{1}{2 \pi \sin h} \int_{C(P, h)} F(Q) d s_{Q} .
\end{aligned}
$$

Hence, under the hypotheses of Theorem 3.9, we have for almost all $P$ in $D$

$$
\Delta_{h} F(P)=\frac{1}{2 \pi} \iint_{I(P, h)} \psi F(Q) \log \left(\cot 2^{-1} P Q \tan 2^{-1} h\right) d Q,
$$

where $C(P, h)+I(P, h) \subset D$.

\section{THE OPERATOR $\Omega$}

4.1. Definition. Put

$$
G(P, Q)=\frac{1}{2 \pi} \log \sin 2^{-1} P Q .
$$

Let $f \in L$ on $S$. Put $f^{+}=\max (f, 0), f^{-}=-\min (f, 0)$. We define

$$
\Omega f(P)=\iint_{S} f(Q) G(P, Q) d Q
$$

at all points $P$ on $S$ at which at least one of the integrals

$$
\iint_{S} f^{+}(Q) G(P, Q) d Q, \quad \iint_{S} f^{-}(Q) G(P, Q) d Q
$$

is finite.

Roughly speaking, the operator $\Omega$ is the inverse of $\psi$. The relation between the two operators is stated more precisely in (4.2.1) and (4.7.2). The special significance of $\Omega$ for our purpose will become evident in Theorem 5.2. It is the purpose of this section to prove the following theorem.

4.2. Theorem. Let $Z$ be a closed set of capacity zero on $S$. Suppose

(i) $F(P)$ is continuous on $S$;

(ii) $\psi^{*} F(P)>-\infty, \psi_{*} F(P)<+\infty$ on $S-Z$; 
(iii) there exists a function $y(P)$, defined on $S, y \in L$ on $S$, such that $y(P)$ $\leqq \psi^{*} F(P)$ on $S$.

Then $\psi F \in L$ on $S$, and we have, at almost all points $P$ of $S$,

$$
F(P)=\Omega \psi F(P)+F_{0},
$$

where $F_{0}$ is the average value of $F$ on $S$.

(More precisely, (4.2.1) holds at all points $P$ at which $\Omega \psi F(P)$ is finitely defined. We do not require this additional result, however.)

In this section it will be convenient to use the notation $f_{0}$ for the average value of the function $f$ on $S$ :

$$
f_{0}=\frac{1}{4 \pi} \iint_{S} f(P) d P .
$$

4.3. Lemma. Let $f \in L$ on $S$. Then $\Omega f \in L$ on $S$ (hence $\Omega f(P)$ is finitely defined almost everywhere on $S$ ), and

$$
\frac{1}{4 \pi} \iint_{S} \Omega f(P) d P=-f_{0}
$$

Proof. We apply Fubini's theorem on the change of order of integration. Observing that

$$
G(P, Q) \leqq 0, \quad \iint_{S} G(P, Q) d P=-1,
$$

we have

$$
\begin{aligned}
\iint_{S} f(Q) d Q & =-\iint_{S} f(Q) d Q \iint_{S} G(P, Q) d P \\
& =-\iint_{S} d P \iint_{S} f(Q) G(P, Q) d Q \\
& =-\iint_{S} \Omega f(P) d P .
\end{aligned}
$$

The lemma follows.

4.4. Lemma. Let $G$ be defined as in (4.1.1). For $P$ on $S$,

$$
\frac{1}{2 \pi \sin h} \int_{C(P, h)} G(M, Q) d s_{M}= \begin{cases}\frac{1}{2 \pi} \log \left(\sin 2^{-1} P Q \cos 2^{-1} h\right) & (P Q>h), \\ \frac{1}{2 \pi} \log \left(\cos 2^{-1} P Q \sin 2^{-1} h\right) & (P Q \leqq h) .\end{cases}
$$


Proof. We choose a coordinate system $(\theta, \phi)$ on $S$, with pole at $P$. Put $P Q=\alpha$; let $M$ be a point on $C(P, h)$. Then

$$
\begin{aligned}
\frac{1}{2 \pi \sin h} \int_{C(P, h)} G(M, Q) d s_{M}= & \frac{1}{2 \pi} \int_{0}^{2 \pi} \frac{1}{2 \pi} \log \sin 2^{-1} M Q d \phi \\
= & \frac{1}{8 \pi^{2}} \int_{0}^{2 \pi} \log (1-\cos h \cos \alpha \\
& -\sin h \sin \alpha \cos \phi) d \phi-\frac{\log 2}{4 \pi} .
\end{aligned}
$$

Applying the formula $[2 ; 348]$

$$
\int_{0}^{2 \pi} \log (a+b \cos x) d x=2 \pi \log \frac{a+\left(a^{2}-b^{2}\right)^{1 / 2}}{2} \quad(a>|b|)
$$

to (4.4.1), the lemma follows.

4.5. Lemma. Let $f \in L$ on $S$. Let $C=C(P, h)$ be a circle on $S$. Then $\Omega f \in L$ on $C$; further, if $\Omega f(P)$ exists, then

$$
\begin{aligned}
\Delta_{h} \Omega f(P)= & \frac{1}{2 \pi} \iint_{I(P, h)} f(Q) \log \left(\cot 2^{-1} P Q \tan 2^{-1} h\right) d Q \\
& +2 f_{0} \log \cos 2^{-1} h .
\end{aligned}
$$

Proof. Since the functions $\log \left(\cos 2^{-1} P Q \sin 2^{-1} h\right)$ and $\log \left(\sin 2^{-1} P Q\right.$ $\left.\cos 2^{-1} h\right)$ are bounded for $Q$ in $I=I(P, h)$ and in $S-I$ respectively, we have, by 4.4 and Fubini's theorem,

$$
\begin{aligned}
\frac{1}{2 \pi} \iint_{I} f(Q) \log \left(\cos 2^{-1} P Q \sin 2^{-1} h\right) d Q \\
\quad+\frac{1}{2 \pi} \iint_{S-I} f(Q) \log \left(\sin 2^{-1} P Q \cos 2^{-1} h\right) d Q \\
=\frac{1}{2 \pi \sin h} \iint_{S} f(Q) d Q \int_{C} G(M, Q) d s_{M} \\
=\frac{1}{2 \pi \sin h} \int_{C} d s_{M} \iint_{S} f(Q) G(M, Q) d Q \\
=\frac{1}{2 \pi \sin h} \int_{C} \Omega f(M) d s_{M} .
\end{aligned}
$$

Hence $\Omega f \in L$ on $C$. Next, assuming that $\Omega f(P)$ exists, we subtract (4.1.2) from (4.5.2), which gives (4.5.1). 
4.6. Definition. Let $f \in L$ on $S$. By $L^{*}(f)$ we mean the set of points $P$ on $S$ at which

$$
\iint_{I(P, h)}|f(Q)-f(P)| d Q=o\left(h^{2}\right) \quad(h \rightarrow 0) .
$$

The set $L^{*}(f)$ is known to include almost all points of $S$. Evidently, if $P \in L^{*}(f)$, then $f(P)$ is finite, and

$$
\begin{aligned}
\iint_{I(P, h)}|f(Q)| d Q & =O\left(h^{2}\right) & (h \rightarrow 0) ; \\
\iint_{I(P, h)} f(Q) d Q & =4 \pi f(P) \sin ^{2} 2^{-1} h+o\left(h^{2}\right) & (h \rightarrow 0) .
\end{aligned}
$$

4.7. Lemma. Let $f \in L$ on $S$. Let $P \in L^{*}(f)$. Then $\Omega f(P)$ exists as a finite number. Furthermore,

$$
\Delta_{h} \Omega f(P)=\frac{1}{2 \pi} \int_{0}^{h} \frac{d \theta}{\sin \theta} \iint_{I(P, \theta)} f(Q) d Q+2 f_{0} \log \cos 2^{-1} h
$$

(4.7.2) $\quad \psi \Omega f(P)=f(P)-f_{0}$.

Proof. Integrating by parts, we have, using (4.6.2),

$$
\begin{aligned}
2 \pi \iint_{S}|f(Q) G(P, Q)| d Q= & \lim _{\boldsymbol{\epsilon} \rightarrow 0} \int_{\epsilon}^{\pi} \log \csc 2^{-1} \theta d \theta \int_{C(P, \theta)}|f(Q)| d s_{Q} \\
= & \lim _{\epsilon \rightarrow 0}\left\{\log \sin 2^{-1} \epsilon \iint_{J(P, \epsilon)}|f(Q)| d Q\right. \\
& \left.+\frac{1}{2} \int_{\epsilon}^{\pi} \cot 2^{-1} \theta d \theta \iint_{I(P, \theta)}|f(Q)| d Q\right\} \\
= & \frac{1}{2} \int_{0}^{\pi} \cot 2^{-1} \theta d \theta \iint_{I(P, \theta)}|f(Q)| d Q .
\end{aligned}
$$

Hence $\Omega f(P)$ is finite. Using (4.5.1) and proceeding as in (4.7.3), we obtain (4.7.1). Substitution of (4.6.3) into (4.7.1) yields (4.7.2).

4.8. Proof of Theorem 4.2. Let $C$ be a circle on $S$ which bounds two domains, $D_{1}$ and $D_{2}$. By Theorem 3.9, $\psi F$ exists p. p. on $S$, and $\in L$ on $D_{1}$ and on $D_{2}$. Hence $\psi F \in L$ on $S$. Put

$$
f(P)=\psi F(P)
$$

at all points $P$ at which $\psi F(P)$ exists. We have to show that

$$
F(P)=\Omega f(P)+F_{0}
$$

(p. p. on $S$ ).

Let $E_{1}$ be the set on which (3.9.4) holds; put $E=E_{1} \cdot L^{*}(f)$. Comparing (3.9.4) and (4.5.1), we see that 


$$
\Delta_{h} F(P)=\Delta_{h} \Omega f(P)-2 f_{0} \log \cos 2^{-1} h
$$

for $P$ on $E$. Hence, by (4.7.1),

$$
\Delta_{h} F(P)=\frac{1}{2 \pi} \int_{0}^{h} \frac{d \theta}{\sin \theta} \iint_{I(P, \theta)} f(Q) d Q \quad(0<h<\pi) .
$$

We now let $h \rightarrow \pi$. The left member of (4.8.4) approaches the limit $F(M)$ $-F(P)$, where $P M$ is a diameter of $S$; the right side diverges if $f_{0} \neq 0$. Hence $f_{0}=0$, and (4.8.3) becomes

$$
\Delta_{h} F(P)=\Delta_{h} \Omega f(P) \quad(P \text { on } E ; 0<h<\pi)
$$

which may be written

$$
2 \pi \sin h(F(P)-\Omega f(P))=\int_{C_{(P, h)}}(F(Q)-\Omega f(Q)) d s_{Q} .
$$

Integrating (4.8.6) with respect to $h$ from 0 to $\pi$, and using (4.3.1), we see that

$$
F(P)-\Omega f(P)=F_{0}+f_{0}=F_{0} \quad(P \text { on } E) .
$$

This proves (4.8.2), and hence the theorem.

It is worth noting that Lemmas 3.4, 3.7, and 3.8 of [6] hold for the operator $\Omega$ as defined in the present paper, if $R$ and $\Lambda$ are replaced by $S$ and $\psi$.

\section{Proof OF UNIQUENESS THEOREMS}

5.1. Lemma. Let $Y_{n}(P)$ be a spherical surface harmonic of degree $n$, $n=0,1,2, \cdots$ Then, for $P$ on $S$,

$$
\psi Y_{n}(P)+n(n+1) Y_{n}(P)=0 .
$$

Furthermore, for $n=1,2,3, \cdots$,

$$
Y_{n}(P)+n(n+1) \Omega Y_{n}(P)=0 .
$$

Proof. Choose a circle $C(P, h)$ on $S$. Using the formula $[1 ; 145]$

$$
Y_{n}(P)=\frac{2 n+1}{4 \pi} \iint_{S} Y_{n}(Q) P_{n}(\cos P Q) d Q \quad(n=0,1,2, \cdots),
$$

we obtain

$$
\begin{aligned}
\int_{C(P, h)} Y_{n}(Q) d s_{Q} & =\frac{2 n+1}{4 \pi} \int_{C(P, h)} d s_{Q} \iint_{S} Y_{n}(M) P_{n}(\cos Q M) d M \\
& =\frac{2 n+1}{4 \pi} \iint_{S} Y_{n}(M) d M \int_{C(P, h)} P_{n}(\cos Q M) d s_{Q}
\end{aligned}
$$

Applying the addition theorem for Legendre polynomials $[1 ; 143]$, we see 
that the last expression in (5.1.4) is equal to

$$
\begin{aligned}
\frac{2 n+1}{4 \pi} \iint_{S} Y_{n}(M) d M \int_{C(P, h)} P_{n}(\cos h) P_{n}(\cos P M) d s_{Q} & \\
& =2 \pi \sin h P_{n}(\cos h) Y_{n}(P) .
\end{aligned}
$$

This, together with (2.1.1), gives

$$
\Delta_{h} Y_{n}(P)=-\left(1-P_{n}(\cos h)\right) Y_{n}(P) .
$$

Hence

$$
\psi Y_{n}(P)=-Y_{n}(P) \lim _{n \rightarrow 0} \frac{1-P_{n}(\cos h)}{\sin ^{2} 2^{-1} h}=-n(n+1) Y_{n}(P),
$$

since $P_{n}^{\prime}(1)=2^{-1} n(n+1)$, as is easily seen from the recursion formula $[1 ; 33]$

$$
(2 n+1) P_{n}(x)=P_{n+1}^{\prime}(x)-P_{n-1}^{\prime}(x) .
$$

Next we note that for $n \geqq 1$ the average value of $Y_{n}$ on $S$ is zero, by (1.1.2). Hence, for $n \geqq 1, \Omega \psi Y_{n}(P)=Y_{n}(P)$, by Theorem 4.2. Applying the operator $\Omega$ to (5.1.1), (5.1.2) is obtained.

It should be pointed out that (5.1.2) can also be proved directly (but more laboriously), without use of Theorem 4.2, by substituting (5.1.3) into (4.1.2).

5.2. TheOREM. Let $f \in L$ on $S$. Then $f(P) \sim \sum_{n=1}^{\infty} Y_{n}(P)$ if and only if $\Omega f(P) \sim-\sum_{n=1}^{\infty} Y_{n}(P) / n(n+1)$. (We refer to (1.2.3) for the notation.)

Proof. We have

$$
\begin{aligned}
\iint_{S} \Omega f(Q) P_{n}(\cos P Q) d Q & =\iint_{S} P_{n}(\cos P Q) d Q \iint_{S} f(M) G(Q, M) d M \\
& =\iint_{S} f(M) d M \iint_{S} P_{n}(\cos P Q) G(Q, M) d Q \\
& =\iint_{S} f(M) \Omega P_{n}(\cos P M) d M \\
& =-\frac{1}{n(n+1)} \iint_{S} f(M) P_{n}(\cos P M) d M .
\end{aligned}
$$

In the last step we applied (5.1.2) to the surface harmonic $P_{n}(\cos P M)$. By (1.2.1), (5.2.1) proves both necessity and sufficiency.

Theorem 5.2 shows that the series $\sum \Omega Y_{n}(P)$ is analogous to a twice integrated trigonometric series (see $[10 ; 274]$ ).

5.3. Proof of Theorem 2.6. We assume without loss of generality that $Y_{0}=0$. Since $F(P)$ satisfies all hypotheses of Theorem $4.2, \psi F(P)$ exists p. p. 
on $S$, that is, the given series is Riemann summable p. p. on $S$. Noting that the average value of $F$ on $S$ is zero, we have, by Theorem 4.2,

$$
F(P)=\Omega \psi F(P) \quad \text { (p. p. on } S \text { ). }
$$

By assumption,

$$
F(P) \sim-\sum_{n=1}^{\infty} Y_{n}(P) / n(n+1)
$$

Hence

$$
\Omega \psi F(P) \sim-\sum_{n=1}^{\infty} Y_{n}(P) / n(n+1),
$$

which, by Theorem 5.2, implies

$$
\psi F(P) \sim \sum_{n=1}^{\infty} Y_{n}(P)
$$

This completes the proof.

In the next lemma, which will be used in the proof of Theorem 2.7, we establish a relation between the Poisson and Riemann sums of a series of spherical surface harmonics; its analogue, for trigonometric series, is due to Rajchman and Zygmund [4;268].

5.4. Lemma. Let $f^{*}(P), f_{*}(P)$ be the upper and lower Poisson sums, and let $\psi^{*} F(P), \psi_{*} F(P)$ be the upper and lower Riemann sums of the series $\sum_{n=1}^{\infty} Y_{n}(P)$, which is of class $K$. Then, for $P$ on $S$, we have

$$
\psi_{*} F(P) \leqq f^{*}(P) ; \quad(\mathrm{b}) \quad f_{*}(P) \leqq \psi^{*} F(P) .
$$

Proof. Let $M$ be a point on $S$. Since $\psi^{*} F$ and $\psi_{*} F$ are not changed if a constant is added to $F$, we may assume without loss of generality that $F(M)$ $=0$. Let $F(r, P), 0 \leqq r<1$, be the function which is harmonic in the interior of $S$ and has $F(P)$ as boundary values. Since $F(P)$ is continuous, we have

$$
\lim _{r \rightarrow 1} F(r, M)=F(M)=0 .
$$

The inequality (a) is trivial if $\psi_{*} F(M)=-\infty$. Hence we need only show that $\psi_{*} F(M)>m$ implies $f^{*}(M) \geqq m$ for any finite number $m$. Consider the function

$$
U(P)=1-\cos M P=1-P_{1}(\cos M P) .
$$

We have $U(M)=0, \psi U(M)=2$ (by (5.1.1)). Adding a multiple of $U(P)$ to $F(P)$, we see that it is sufficient to prove that the inequality

$$
\psi_{*} F(M)>0
$$


implies

$$
f^{*}(M) \geqq 0
$$

Put

$$
f(r, P)=\sum_{n=1}^{\infty} Y_{n}(P) r^{n} \quad(0 \leqq r<1) .
$$

It follows from Laplace's equation

$$
\frac{1}{\sin \theta} \frac{\partial}{\partial \theta}\left(\sin \theta \frac{\partial F}{\partial \theta}\right)+\frac{1}{\sin ^{2} \theta} \frac{\partial^{2} F}{\partial \phi^{2}}+\frac{\partial}{\partial r}\left(r^{2} \frac{\partial F}{\partial r}\right)=0
$$

that

$$
f(r, P)+\frac{\partial}{\partial r}\left(r^{2} \frac{\partial F}{\partial r}\right)=0 .
$$

We assume that (5.4.2) holds. If (5.4.3) is false, then (5.4.4) implies

$$
\left(r^{2} F^{\prime}(r, M)\right)^{\prime}>0
$$

$\left(0 \leqq r_{0} \leqq r<1\right)$,

where $r_{0}$ is constant, and the primes denote differentiation with respect to $r$. By the mean value theorem, and (5.4.1), there exists $s, r<s<1$, such that

$$
\frac{r F(r, M)}{1-r}=\frac{F(M)-F(r, M)}{1-1 / r}=\frac{F^{\prime}(s, M)}{(1 / s)^{\prime}}=-s^{2} F^{\prime}(s, M) .
$$

Hence there exists $t, s<t<1$, such that

$$
\begin{aligned}
\frac{r F(r, M)}{1-r} & -\frac{s F(s, M)}{1-s} \\
& =t^{2} F^{\prime}(t, M)-s^{2} F^{\prime}(s, M) .
\end{aligned}
$$

This expression is positive, for $r \geqq r_{0}$, by (5.4.5). To obtain a contradiction, it is now enough to show that (5.4.2) implies

$$
\liminf _{r \rightarrow 1} \frac{d}{d r}\left\{\frac{r F(r, M)}{1-r}\right\}>0 .
$$

Let $M$ be the pole of a spherical coordinate system $(\theta, \phi)$. The Poisson formula

$$
F(r, M)=\frac{1}{4 \pi} \int_{0}^{\pi} \int_{0}^{2 \pi} \frac{1-r^{2}}{\Delta^{3}} F(\theta, \phi) \sin \theta d \theta d \phi
$$

where

$$
\Delta^{2}=1-2 r \cos \theta+r^{2}
$$


may be differentiated under the integral sign. We thus obtain

$$
\frac{d}{d r}\left\{\frac{r F(r, M)}{1-r}\right\}=\frac{1}{4 \pi} \int_{0}^{\pi} \int_{0}^{2 \pi} \frac{\partial}{\partial r}\left\{\frac{r(1+r)}{\Delta^{3}}\right\} F(\theta, \phi) \sin \theta d \theta d \phi .
$$

Since

$$
\frac{\partial}{\partial r}\left\{\frac{r(1+r)}{\Delta^{3}}\right\}=\frac{(1-r)\left(1+r(3+\cos \theta)+r^{2}\right)}{\Delta^{5}},
$$

we see that

$$
\lim _{r \rightarrow 1} \frac{\partial}{\partial r}\left\{\frac{r(1+r)}{\Delta^{3}}\right\}=0
$$

uniformly for $0<\epsilon \leqq \theta \leqq \pi$. By (5.4.2) there exists $\epsilon>0$ and $h>0$ such that

$$
\frac{1}{2 \pi} \int_{0}^{2 \pi} F(\theta, \phi) d \phi \geqq 2 h \sin ^{2} 2^{-1} \theta \quad(0<\theta \leqq \epsilon) .
$$

Since (5.4.7) is non-negative, (5.4.8) and (5.4.9) imply

$$
\begin{aligned}
\underset{r \rightarrow 1}{\lim \inf } \frac{d}{d r}\left\{\frac{r F(r, M)}{1-r}\right\} & \\
& =\liminf _{r \rightarrow 1} \frac{1}{4 \pi} \int_{0}^{\epsilon} \frac{\partial}{\partial r}\left\{\frac{r(1+r)}{\Delta^{3}}\right\} \sin \theta d \theta \int_{0}^{2 \pi} F(\theta, \phi) d \phi \\
& \geqq h \liminf _{r \rightarrow 1}^{\epsilon} \int_{0}^{\epsilon} \frac{\partial}{\partial r}\left\{\frac{r(1+r)}{\Delta^{3}}\right\} \sin ^{2} 2^{-1} \theta \sin \theta d \theta \\
& =h \lim _{r \rightarrow 1} \frac{1}{4 \pi} \int_{0}^{\epsilon} \frac{\partial}{\partial r}\left\{\frac{r(1+r)}{\Delta^{3}}\right\}(1-\cos \theta) \sin \theta d \theta \int_{0}^{2 \pi} d \phi \\
& =h \lim _{r \rightarrow 1} \frac{d}{d r}\left\{\frac{r}{1-r} \cdot \frac{1}{4 \pi} \int_{0}^{\pi} \int_{0}^{2 \pi} \frac{1-r^{2}}{\Delta^{3}}\left(1-P_{1}(\cos \theta)\right) \sin \theta d \theta d \phi\right\} \\
& =h \lim _{r \rightarrow 1} \frac{d}{d r}\left\{\frac{r}{1-r}\left(1-P_{1}(1) r\right)\right\} \\
& =h>0 .
\end{aligned}
$$

This proves (5.4.6), and hence part (a) of the lemma. The inequality (b) follows by a change of sign.

5.5. Proof of Theorem 2.7. We assume without loss of generality that $Y_{0}=0$. By (i) there exists a continuous function $F(P)$ such that

$$
F(P) \sim-\sum_{n=1}^{\infty} Y_{n}(P) / n(n+1)
$$


By (ii) and (iii), Lemma 5.4 implies

$$
\begin{array}{lr}
\psi^{*} F(P) \geqq f_{*}(P)>-\infty & (P \text { on } S-Z), \\
\psi_{*} F(P) \leqq f^{*}(P)<+\infty & (P \text { on } S-Z), \\
\psi^{*} F(P) \geqq f_{*}(P) \geqq y(P) & (P \text { on } S) .
\end{array}
$$

We see that all requirements of Theorem 2.6 are satisfied. Hence

$$
\psi F(P) \sim \sum_{n=1}^{\infty} Y_{n}(P) .
$$

The given series has now been shown to be a Laplace series. Hence it is Poisson summable p. p. on $S$, and is the Laplace series of its Poisson sum [1; 353].

This completes the proof. Since the assumptions of 2.7 imply those of 2.6, we note that Theorem 2.6 has greater generality than Theorem 2.7.

\section{APPENDIX}

So far we have considered only series of class $K$. The series of associated Legendre functions $\sum_{n=1}^{\infty}(2 n+1) P_{n}^{1}(\cos \theta) \sin \phi$, which is Poisson summable to zero at all points of $S$, shows that no condition imposed on the Poisson sums alone can enable us to infer that the given series is a Laplace series. The question arises as to whether condition (i) can be omitted in 2.7 if the given series is assumed to converge. We do not answer this question here, but will show:

6.1. ThEOREM. There exist series of spherical surface harmonics which converge at every point of $S$, without being of class $K$.

We define the modulus $c_{n}$ of the spherical surface harmonic $Y_{n}$ by

$$
c_{n}^{2}=\frac{1}{4 \pi} \iint_{S} Y_{n}^{2}(P) d P .
$$

If $f \in L^{2}$ on $S$, and if $f \sim \sum Y_{n}(P)$, then it follows from Bessel's inequality that $c_{n}=o(1)$. Hence a necessary condition for $\sum Y_{n}(P)$ to be of class $K$ is that $c_{n}=o\left(n^{2}\right)$. Thus the following result, which is in marked contrast to the Cantor-Lebesgue theorem for trigonometric series, implies 6.1.

6.2. Theorem. Given any real number $m$, there exists a series $\sum Y_{n}(P)$ which converges at every point $P$ of $S$, and for which $n^{-m} c_{n} \rightarrow \infty$ as $n \rightarrow \infty$.

Proof. The series

$$
\sum_{n=1}^{\infty} Y_{n}(P)=\sum_{n=1}^{\infty} Y_{n}(\theta, \phi)=\sum_{n=1}^{\infty} n^{m+1} \sin ^{n-1} \theta \cos \theta \sin (n-1) \phi,
$$

where $(\theta, \phi)$ is a spherical coordinate system on $S$, has the desired properties. 


\section{BIBLIOGRAPHY}

1. E. W. Hobson, The theory of spherical and ellipsoidal harmonics, Cambridge University Press, 1931.

2. O. D. Kellogg, Foundations of potential theory, Berlin, Springer, 1929.

3. M. Plancherel, Les problèmes de Cantor et de du Bois Reymond dans la théorie de séries de polynômes de Legendre, Ann. École Norm. Sup. vol. 31 (1914) pp. 223-262.

4. A. Rajchman and A. Zygmund, Sur la possibilité d'appliquer la méthode de Riemann aux séries trigonométriques sommables par le procédé de Poisson, Math. Zeit. vol. 25 (1926) pp. 261273.

5. F. Riesz, Sur les fonctions subharmoniques et leur rappert à la théorie du potential, Acta Math. vol. 48 (1926) pp. 329-343.

6. W. Rudin, Integral representation of continuous functions, Trans. Amer. Math. Soc. vol. 68 (1950) pp. 279-287.

7. S. Verblunsky, On the theory of trigonometric series (II), Proc. London Math. Soc. vol. 34 (1932) pp. 457-491.

8. A. Zygmund, Sur les séries trigonométriques sommables par le procédé de Poisson, Math. Zeit. vol. 25 (1926) pp. 274-290.

9. - Sur la théorie riemannienne de certains systèmes orthogonaux I, Studia Mathematica vol. 2 (1930) pp. 97-170.

10. - Trigonometrical series, Warsaw, 1935.

DUKE UNIVERSITY, Durham, N. C. 